TITLE:

\title{
Numerical Analysis of Nonlinear Acoustic Wave Propagation in a Rarefied Gas
}

\author{
$\operatorname{AUTHOR}(\mathrm{S})$ : \\ Tsuji, Tetsuro; Aoki, Kazuo
}

\section{CITATION:}

Tsuji, Tetsuro ...[et al]. Numerical Analysis of Nonlinear Acoustic Wave Propagation in a Rarefied Gas. AIP Conference Proceedings: 28th International Symposium on Rarefied Gas Dynamics 2012 2012, 1501: 115-122

ISSUE DATE:

2012-11-27

URL:

http://hdl.handle.net/2433/187984

\section{RIGHT:}

(c) 2012 American Institute of Physics. This article may be downloaded for personal use only. Any other use requires prior permission of the author and the American Institute of Physics. 


\title{
Numerical Analysis of Nonlinear Acoustic Wave Propagation in a Rarefied Gas
}

\author{
Tetsuro Tsuji ${ }^{\dagger}$ and Kazuo Aoki ${ }^{\dagger, 末}$ \\ ${ }^{\dagger}$ Department of Mechanical Engineering and Science, Kyoto University, Kyoto 606-8501, Japan \\ $¥$ Advanced Research Institute of Fluid Science and Engineering, Kyoto University, Kyoto 606-8501, Japan
}

\begin{abstract}
Unsteady motion of a rarefied gas in a half space, caused by an infinitely wide plate when it starts a longitudinal and harmonic oscillation, is investigated numerically on the basis of the Bhatnagar-Gross-Krook (BGK) model of the Boltzmann equation. A deterministic method capable of describing the singularities in the molecular velocity distribution function produced by the oscillating plate, which was developed recently by the authors, is used as a solution method, and the unsteady behavior of the gas is obtained accurately. The streaming motion and the attenuation of the wave, observed in the existing work using the direct simulation Monte Carlo (DSMC) method (T. Ohwada and M. Kunihisa, in Rarefied Gas Dynamics, AIP, Melville, 2003, pp. 202-209), are also obtained. In addition, some pieces of numerical evidence that clarify the long-time behavior of the gas are provided. For example, one-period averages of the momentum and energy fluxes across the oscillating plate tend to approach their values for a periodic state (a constant for the momentum flux and zero for the energy flux) slowly, the rate of approach being likely to be inversely proportional to the square root of time.
\end{abstract}

Keywords: Rarefied gas dynamics, Nonlinear acoustic wave, Moving-boundary problems

PACS: $47.45 .-\mathrm{n}, 47.45 . \mathrm{Ab}, 43.25 .-\mathrm{x}, 51.40 .+\mathrm{p}$

\section{INTRODUCTION}

Acoustic wave propagation in a gas is one of the fundamental problems in gas dynamics. Gas motion in a semi-infinite space induced by an infinitely wide plate oscillating longitudinally is a typical and the simplest setting, for which the linearized Euler equation gives the plane-wave solution (see e.g., [1]).

However, when the frequency of oscillation of the plate is high, or when the gas under consideration is rarefied, the frequency of oscillation can be comparable to the collision frequency of the gas molecules. In such cases, the continuum gas dynamics is not applicable any more and should be replaced by kinetic theory (see e.g., [2]). In fact, this is a classical subject in rarefied gas dynamics and has been investigated by many authors (e.g., [3, 4, 5, 6, 7, 8, 9, 10]). More specifically, the acoustic wave propagation in a half space was studied using linearized kinetic equations, under the assumption that the speed of the oscillating plate is much lower than the sonic speed, and it was reported that the effect of gas rarefaction (or the non-continuum effect) led to attenuation of the propagating wave and spatial inhomogeneity of its wave length. However, when the amplitude of oscillation is increased at a fixed frequency, or when the frequency is increased at a fixed amplitude, the speed of oscillation of the plate may become comparable to the sonic speed, so that the nonlinearity cannot be neglected. In the present study, we consider such cases with full nonlinearity. More precisely, we investigate unsteady motion in a semi-infinite expanse of a rarefied gas caused by an infinitely wide plate when it starts a longitudinal harmonic oscillation. We carry out accurate numerical analysis using the Bhatnagar-Gross-Krook (BGK) model $[11,12]$ of the Boltzmann equation and give precise description of time-evolution of the macroscopic quantities.

The same nonlinear problem was investigated using classical gas dynamics (the Euler equation) in [13, 14], where the formation of sawtooth-like waves and the streaming motion toward infinity, accompanied by a rarefaction region, were found and attributed to the effect of nonlinearity. In particular, with the help of a long-time computation (up to about 190 periods of oscillation), it was pointed out that a rarefaction region develops near the oscillating plate and extends linearly in time and that a quasi-steady periodic state is formed near the plate. On the other hand, a similar nonlinear problem, i.e., wave propagation caused by a sudden start of a sawtooth oscillation of a plate, was studied for a rarefied gas by the direct simulation Monte Carlo (DSMC) method in [15], where both the effect of gas rarefaction and that of nonlinearity mentioned above were observed. In the present study, we expect to observe more or less the same phenomena, but an emphasis will be put on the quantitative description of the long-time behavior.

Finally, we mention another aspect of the present study. In most of the works based on the linearized kinetic equations mentioned above, the oscillating plate is approximated by a stationary wall, and the oscillation is produced 
by imposing an oscillatory macroscopic velocity in the boundary condition. In the present nonlinear problem, however, such an approximation is not valid, so that we have to handle the problem with a moving boundary. It is a challenging subject in kinetic theory because the oscillating plate continuously sends discontinuities in the molecular velocity distribution function in the gas. We tackle this problem by a deterministic solution method, capable of describing the discontinuities caused by a moving plate, that was developed recently by the authors [20].

\section{FORMULATION OF THE PROBLEM}

\section{Problem and Assumptions}

We consider a semi-infinite expanse of an ideal monatomic gas in a uniform equilibrium state at rest with density $\rho_{0 *}$ and temperature $T_{0 *}$, bounded by an infinitely wide plate kept at uniform and constant temperature $T_{0 *}$. The plate is placed at $X_{1}=a_{*}(>0)$, where $X_{i}(i=1,2,3)$ denotes the Cartesian coordinate system with the $X_{1}$ axis perpendicular to the plate. At time $t_{*}=0$, the plate starts an oscillation around $X_{1}=0$ with angular frequency $\omega_{*}$ according to $X_{1}=X_{w}\left(t_{*}\right)$ with $X_{w}\left(t_{*}\right)=a_{*} \cos \omega_{*} t_{*}$. We investigate the subsequent unsteady motion of the gas numerically on the basis of kinetic theory when the frequency of oscillation of the plate is comparable to the mean collision frequency of gas molecules.

The assumptions for our analysis are as follows:

1. The behavior of the gas is described by the BGK model of the Boltzmann equation ([11, 12]; see also [2]).

2. The gas molecules undergo diffuse reflection on the plate (see, e.g., [2]). More specifically, the molecules reflected on the plate are distributed according to the half-range Maxwellian distribution characterized by the velocity and temperature of the plate and with the density adjusted in such a way that there is no net mass flow across the boundary.

3. The gas motion is one dimensional, that is, the motion is only in the $X_{1}$ direction, and the physical quantities do not depend on $X_{2}$ and $X_{3}$.

Before formulating the problem, we summarize the notations used throughout the present paper. We first introduce (and repeat) basic dimensional quantities, that is, $t_{*}$ is the time variable, $X_{i}$ the Cartesian coordinate system in space, $\xi_{i}$ the molecular velocity, $X_{w}$ the position $\left(X_{1}\right.$ coordinate) of the plate, $f_{*}$ the velocity distribution function of gas molecules, $\rho_{*}$ the density, $\left(u_{1 *}, 0,0\right)$ the flow velocity, $T_{*}$ the temperature, $p_{*}$ the pressure, $p_{11 *}$ the $X_{1}-X_{1}$ component of the stress tensor (normal stress), and $\left(q_{1 *}, 0,0\right)$ the heat-flow vector. Then, adopting the time and length scales given respectively by

$$
1 / \omega_{*}, \quad c_{0 *} / \omega_{*},
$$

where $c_{0 *}=\left(2 R T_{0 *}\right)^{1 / 2}$ with $R$ the gas constant per unit mass, we introduce the dimensionless counterparts, $t, x_{i}, \zeta_{i}$, $x_{w}, f, \rho, u_{1}, T, p, p_{11}$, and $q_{1}$, as follows:

$$
\begin{aligned}
& t=t_{*} /\left(1 / \omega_{*}\right), \quad x_{i}=X_{i} /\left(c_{0 *} / \omega_{*}\right), \quad \zeta_{i}=\xi_{i} / c_{0 *}, \quad x_{w}=X_{w} /\left(c_{0 *} / \omega_{*}\right), \quad f=f_{*} /\left(\rho_{0 *} c_{0 *}^{3}\right), \\
& \rho=\rho_{*} / \rho_{0 *}, \quad u_{1}=u_{1 *} / c_{0 *}, \quad T=T_{*} / T_{0 *}, \quad p=p_{*} / p_{0 *}, \quad p_{11}=p_{11 *} / p_{0 *}, \quad q_{1}=q_{1 *} /\left(p_{0 *} c_{0 *}\right),
\end{aligned}
$$

where $p_{0 *}=R \rho_{0 *} T_{0 *}$. In addition, we define the Knudsen number Kn and the Mach number Ma as

$$
\mathrm{Kn}=l_{0 *} /\left(c_{0 *} / \omega_{*}\right), \quad \mathrm{Ma}=a_{*} \omega_{*} /\left(5 R T_{0 *} / 3\right)^{1 / 2},
$$

where $l_{0 *}$ is the mean free path of the gas molecules at the reference equilibrium state at rest, $a_{*} \omega_{*}$ is the maximum speed of the oscillating plate, and $\left(5 R T_{0 *} / 3\right)^{1 / 2}$ is the sonic speed at temperature $T_{0 *}$; the $l_{0 *}$ is defined in terms of the collision frequency $v_{0 *}$ of the gas molecules at the reference state as $l_{0 *}=\left(2 / \pi^{1 / 2}\right)\left(c_{0 *} / v_{0 *}\right)$; for the BGK model, $v_{0 *}$ is given as $v_{0 *}=A_{c} \rho_{0 *}$ with a constant $A_{c}$.

\section{Basic Equations}

The independent variables (i.e., the arguments of $f$ ) in the present spatially one-dimensional problem are $x_{1}, \zeta_{1}, \zeta_{2}$, $\zeta_{3}$, and $t$. However, in the case of the BGK model, one can eliminate $\zeta_{2}$ and $\zeta_{3}$ (the molecular velocity components parallel to the plate) by introducing the following marginal velocity distribution functions $g$ and $h$ [16]:

$$
\left[\begin{array}{l}
g\left(x_{1}, \zeta_{1}, t\right) \\
h\left(x_{1}, \zeta_{1}, t\right)
\end{array}\right]=\int_{-\infty}^{\infty} \int_{-\infty}^{\infty}\left[\begin{array}{c}
1 \\
\zeta_{2}^{2}+\zeta_{3}^{2}
\end{array}\right] f\left(x_{1}, \zeta_{1}, \zeta_{2}, \zeta_{3}, t\right) \mathrm{d} \zeta_{2} \mathrm{~d} \zeta_{3} .
$$


To be more specific, the BGK model equation for $f$ is reduced to simultaneous integro-differential equations for $g$ and $h$, which read as

$$
\begin{aligned}
& \frac{\partial \Phi}{\partial t}+\zeta_{1} \frac{\partial \Phi}{\partial x_{1}}=\frac{2}{\sqrt{\pi}} \frac{1}{\mathrm{Kn}} \rho(\lambda M-\Phi), \quad\left(\Phi=\left[\begin{array}{l}
g \\
h
\end{array}\right], \lambda=\left[\begin{array}{c}
1 \\
T
\end{array}\right]\right), \\
& M=\frac{\rho}{(\pi T)^{1 / 2}} \exp \left(-\frac{\left(\zeta_{1}-u_{1}\right)^{2}}{T}\right), \\
& \rho=\int_{-\infty}^{\infty} g \mathrm{~d} \zeta_{1}, \quad u_{1}=\frac{1}{\rho} \int_{-\infty}^{\infty} \zeta_{1} g \mathrm{~d} \zeta_{1}, \quad T=\frac{2}{3 \rho} \int_{-\infty}^{\infty}\left[\left(\zeta_{1}-u_{1}\right)^{2} g+h\right] \mathrm{d} \zeta_{1} .
\end{aligned}
$$

The initial condition for Eq. (5) is given by

$$
g=h=M_{0}, \quad M_{0}=\pi^{-1 / 2} \exp \left(-\zeta_{1}^{2}\right), \quad \text { at } t=0 .
$$

The boundary condition on the oscillating plate is written as

$$
\begin{aligned}
& g\left(x_{1}, \zeta_{1}, t\right)=h\left(x_{1}, \zeta_{1}, t\right)=M_{w}\left(\zeta_{1}, t\right), \quad \text { for } \zeta_{1}-v_{w}(t)>0, \text { at } x_{1}=x_{w}(t), \\
& M_{w}\left(\zeta_{1}, t\right)=\pi^{-1 / 2} \sigma_{w}(t) \exp \left(-\left[\zeta_{1}-v_{w}(t)\right]^{2}\right), \\
& \sigma_{w}(t)=-2 \sqrt{\pi} \int_{\zeta_{1}-v_{w}(t)<0}\left[\zeta_{1}-v_{w}(t)\right] g\left(x_{w}(t), \zeta_{1}, t\right) \mathrm{d} \zeta_{1},
\end{aligned}
$$

where

$$
x_{w}(t)=\sqrt{5 / 6} \mathrm{Ma} \cos t, \quad v_{w}(t)=\mathrm{d} x_{w}(t) / \mathrm{d} t=-\sqrt{5 / 6} \mathrm{Ma} \sin t,
$$

that is, $c_{0 *} v_{w}$ is the dimensional velocity of the plate.

As is seen from Eqs. (5)-(7), the present problem is characterized by the two parameters: Kn and Ma. In place of these, we use

$$
\mathrm{K}=(\sqrt{\pi} / 2) \mathrm{Kn}, \quad \mathrm{M}=\sqrt{5 / 6} \mathrm{Ma}
$$

in the following.

The dimensionless pressure $p$ is given by the equation of state, and the dimensionless normal stress $p_{11}$ and heat flux $q_{1}$ are expressed in terms of the marginals $g$ and $h$, i.e.,

$$
p=\rho T, \quad p_{11}=2 \int_{-\infty}^{\infty}\left(\zeta_{1}-u_{1}\right)^{2} g \mathrm{~d} \zeta_{1}, \quad q_{1}=\int_{-\infty}^{\infty}\left(\zeta_{1}-u_{1}\right)\left[\left(\zeta_{1}-u_{1}\right)^{2} g+h\right] \mathrm{d} \zeta_{1} .
$$

We also denote by $\mathscr{P}$ and $\mathscr{E}$, respectively, the $X_{1}$ component of the momentum and the energy, per unit area and time, transferred to the gas from the oscillating plate. Then, they are expressed as

$$
\mathscr{P}=\left.p_{11}\right|_{x_{1}=x_{w}(t)}, \quad \mathscr{E}=\left.\left[q_{1}+p_{11} v_{w}(t)\right]\right|_{x_{1}=x_{w}(t)} .
$$

\section{Remarks on Numerical Method}

As is well known, the molecular velocity distribution function on the plane boundary is generally discontinuous for the molecular velocity tangential to the boundary (see [2]). Unlike the case of convex boundary (here, "convex" means convex toward the gas), the discontinuity does not propagate into the gas if the plane boundary is at rest or moving at a constant velocity (when no external force acts on the gas molecules), since the characteristic lines of the Boltzmann (or BGK) equation for the molecular velocity equal to the velocity of the boundary stay on the plane. However, when the boundary is accelerated in the direction opposite to the side of the gas, the tangential characteristic lines go into the domain of the gas as time proceeds, so that the molecular velocity distribution function becomes discontinuous in the gas. It decays in time and space because of intermolecular collisions, as in the case of the discontinuity caused by a convex body in steady problems $([2,17])$.

In the present problem, the oscillating plate sends the discontinuities and other types of weaker singularities continuously in the gas (see $[18,19])$. In order to obtain an accurate numerical solution, especially near the plate, by a deterministic method, we have to treat the discontinuities carefully. Recently we have developed a method, which is basically a method of characteristics, that is capable of describing the propagation of discontinuities, together with other types of weaker singularities, caused by the oscillating plate [20]. This method is used in the present study (its explanation is omitted here because of limited space). 

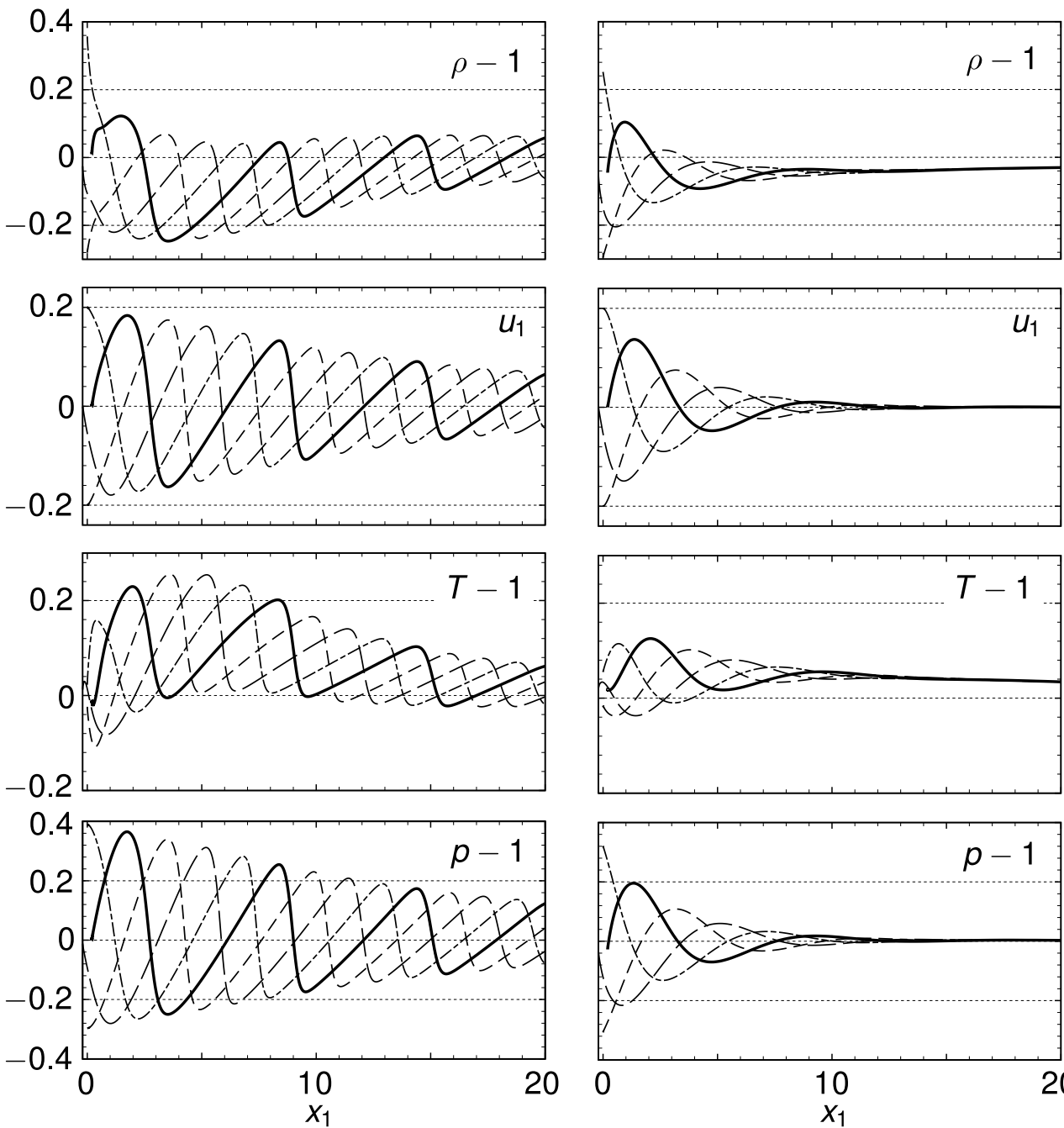

(a) $\mathrm{K}=0.05$

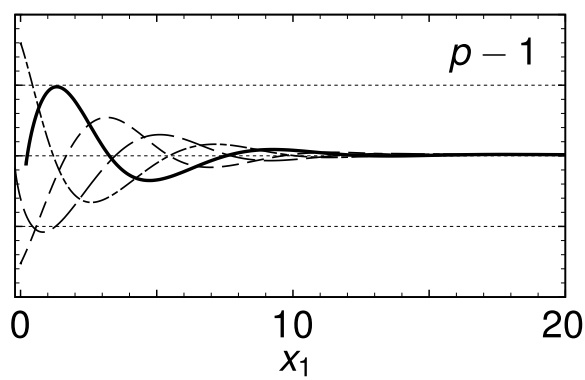

(b) $\mathrm{K}=2$

FIGURE 1. Profiles of $\rho, u_{1}, T$, and $p$ for $\mathrm{M}=0.2$. (a) $\mathrm{K}=0.05$, (b) $\mathrm{K}=2$. In (a), the profiles at $t / 2 \pi=19.25$ (dashed), 19.5 (long-dashed), 19.75 (dash-dotted), and 20 (solid) are shown, and in (b), those at $t / 2 \pi=79.25$ (dashed), 79.5 (long-dashed), 79.75 (dash-dotted), and 80 (solid) are shown.

\section{RESULT AND DISCUSSIONS}

In the present paper, we show the results only for $\mathrm{M}=0.2$ and for some different values of $\mathrm{K}$. We restrict ourselves to the macroscopic quantities, leaving the detailed description of the molecular velocity distribution function of complex shape in the forthcoming paper [20].

\section{Unsteady Behavior of Macroscopic Quantities}

In Fig. 1, we show the profiles of the density $\rho$, flow velocity $u_{1}$, temperature $T$, and pressure $p$ over a few wave lengths during one period for $(\mathrm{K}, \mathrm{M})=(0.05,0.2)$ [panel (a)] and $(2,0.2)$ [panel (b)]. In panel (a), the profiles are shown at $t / 2 \pi=19.25,19.5,19.75$, and 20 , whereas in panel (b), they are shown at $t / 2 \pi=79.25,79.5,79.75$, and 


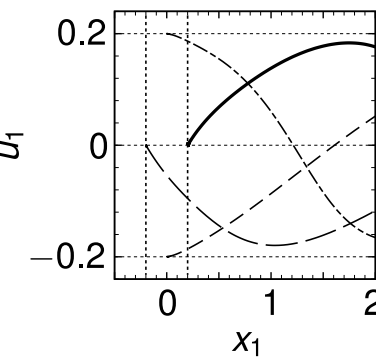

(a) $\mathrm{K}=0.05$

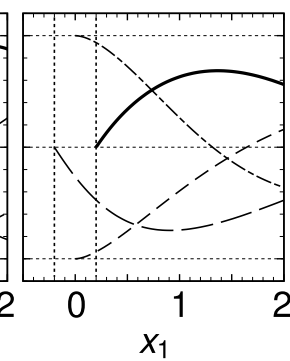

(b) $\mathrm{K}=2$

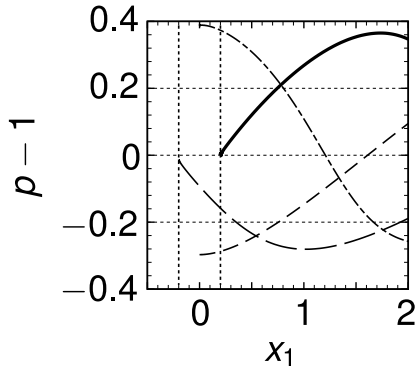

(c) $\mathrm{K}=0.05$

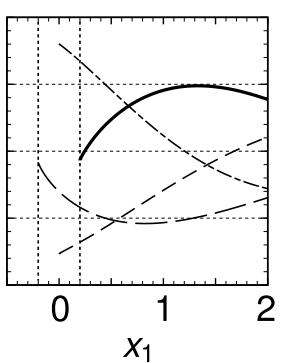

(d) $\mathrm{K}=2$

FIGURE 2. Profiles of $u_{1}$ and $p$ for $\mathrm{M}=0.2$ near the oscillating plate (closeup of Fig. 1). (a) $u_{1}$ at $\mathrm{K}=0.05$, (b) $u_{1}$ at $\mathrm{K}=2$, (c) $p$ at $\mathrm{K}=0.05$, (d) $p$ at $\mathrm{K}=2$. The two vertical dotted lines indicate the range of the plate motion, i.e., $x_{1} \in[-\mathrm{M}, \mathrm{M}]$. In (a) and (c), the profiles at $t / 2 \pi=19.25$ (dashed), 19.5 (long-dashed), 19.75 (dash-dotted), and 20 (solid) are shown, and in (b) and (d), those at $t / 2 \pi=79.25$ (dashed), 79.5 (long-dashed), 79.75 (dash-dotted), and 80 (solid) are shown.

80. In both panels, one can see the time evolution by following the four different types of lines in the order of the dashed line, the long-dashed line, the dash-dotted line, and the solid line. Figure 2 is a close-up of Fig. 1 near the plate for $u_{1}$ and $p$; panels (a) $(\mathrm{K}=0.05)$ and (b) $(\mathrm{K}=2)$ are for $u_{1}$, and panels (c) $(\mathrm{K}=0.05)$ and (d) $(\mathrm{K}=2)$ are for $p$.

Since the Knudsen number is small $(K=0.05)$ in Fig. 1(a), a sawtooth-like wave is observed as in the case of nonlinear acoustic wave based on the Euler equation $[13,14]$. Such a wave is also observed in the DSMC simulation [15]. In Fig. 1(b), where the Knudsen number is intermediate $(K=2)$, the wave attenuates quickly, and the sawtooth like profile is not observed. The amplitude of the wave near the plate is smaller for larger K, as seen from Fig. 2. The strong attenuation of the wave at intermediate Knudsen numbers is also reported in the previous studies based on linearized kinetic equations.

\section{One-period Averages of Macroscopic Quantities}

Let $h\left(x_{1}, t\right)$ stand for the macroscopic quantities, i.e., $h=\rho, u_{1}, T$, etc., and $\bar{h}\left(x_{1}, t\right)$ be its time average over one period from $t-2 \pi$ to $t$, i.e.,

$$
\bar{h}\left(x_{1}, t\right)=\frac{1}{2 \pi} \int_{t-2 \pi}^{t} h\left(x_{1}, s\right) \mathrm{d} s .
$$

We show in Fig. 3 the time evolution of the profiles of the one-period averages, $\bar{\rho}, \bar{u}_{1}$, and $\bar{T}$, for $\mathrm{M}=0.2$ and $\mathrm{K}=2$ : panels (a) and (d) are for $\bar{\rho}$, panels (b) and (e) for $\bar{u}_{1}$, and panels (c) and (f) for $\bar{T}$. In the figure, the profiles at $t / 2 \pi=20$ (dashed line), 30, 40, .., 70 (solid line for all), and 80 (bold line) are shown, and panels (a), (b), (c) show the profiles in the near field. In Fig. 3(e), the small circle indicates the profile of the one-period average of the mass flux $\overline{\rho u_{1}}$. It is seen from Fig. 3 that a weak compression wave, decreasing very slowly, propagates toward infinity, and a high-temperature (low-density) region is developed slowly near the plate. The former wave is called the streaming motion [13, 14, 15], since the one-period average of the mass flux is positive, i.e., there is a net mass flux toward infinity, behind the wave front [see Fig. 3(e)]. The average flux $\overline{\rho u_{1}}$ is almost the same as $\bar{u}_{1}$ in the far field because the deviation of $\rho$ from 1 is small there. Note that $\overline{\rho u_{1}}$ is almost constant in time in the far field in the case of the Euler equations (see Fig. 9 in [14]). We note here that the behavior shown in Fig. 3 is qualitatively similar to that in the unsteady rarefied gas flow in a half space caused by sudden heating of the stationary plate [21] (or Chap. 4.8 in [2]) though the present computation up to $t / 2 \pi=80$ is not long enough to compare with the long-time asymptotes in [21]. In the latter problem, the heating of the gas is caused by the energy supply by the heated plate, which eventually vanishes in the long-time limit. In the present problem, the corresponding energy supply is provided by the oscillation of the plate, and it should also vanish in the long-time limit in which a steady oscillation is established in the near field.

Now we give a close look at the development of the high-temperature region. Let $x_{1}=x_{0.02}(t)$ be the position at time $t$ of the point at which $\bar{T}\left(x_{1}, t\right)-1=0.02$ holds [see Fig. 3(c); if there are more than one such points, we take the largest one]. Figures 4(a), 4(b), and 4(c) show, respectively, $x_{0.02} \mathrm{vs} t, \mathrm{~d} x_{0.02} / \mathrm{d} t$ vs $t$ (in the double-logarithmic scale $)$, and $\alpha\left(\mathrm{d} x_{0.02} / \mathrm{d} t\right)$ vs $t$ at some different $\mathrm{K}$ for $\mathrm{M}=0.2$, where $\alpha\left(\mathrm{d} x_{0.02} / \mathrm{d} t\right)\left[=\mathrm{d} \log _{10}\left(\mathrm{~d} x_{0.02} / \mathrm{d} t\right) / \mathrm{d} \log 10 t\right]$ is 

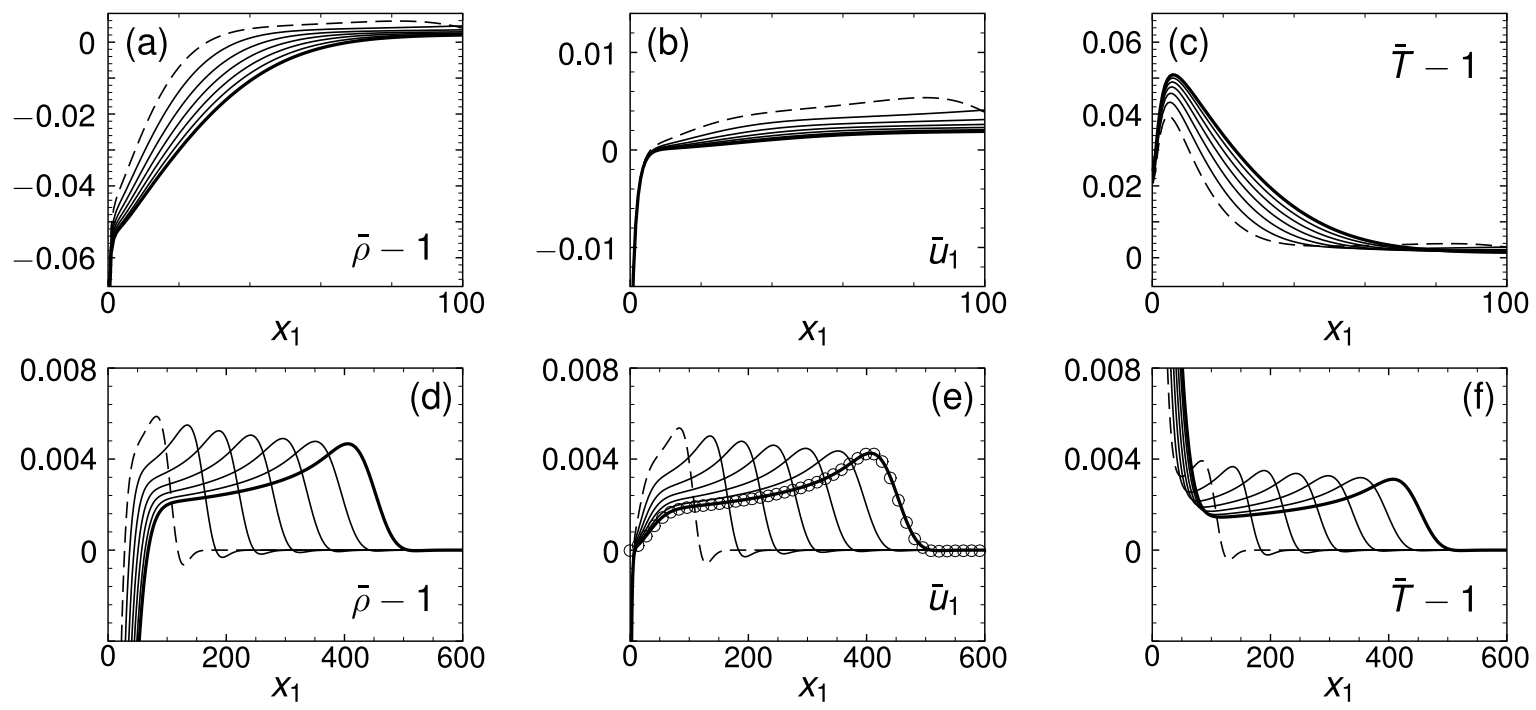

FIGURE 3. Profiles of the one-period averages $\bar{\rho}, \bar{u}_{1}$, and $\bar{T}$ for $\mathrm{M}=0.2$ and $\mathrm{K}=2$. (a), (d) $\bar{\rho}$; (b), (e) $\bar{u}_{1}$; (c), (f) $\bar{T}$. The profiles at $t / 2 \pi=20$ (dashed), 30, $\cdots, 70$ (solid for all) and 80 (bold) are shown. In (a), (b), (c), the region $x_{1} \in[0,100]$ is shown, and in (d), (e), (f), the region $x_{1} \in[0,600]$ is shown with a magnified vertical scale. In (e), $\overline{\rho u_{1}}$ at $t / 2 \pi=80$ is also shown by small circles.

the gradient of a curve in panel (b). The curves in panel (c) exhibit small fluctuations because they are essentially the second derivatives of the curve in panel (a). Nevertheless, they tend to approach $-1 / 2$. Therefore, for each $\mathrm{K}$, the velocity of the point, $\mathrm{d} x_{0.02} / \mathrm{d} t$, tends to approach a straight line with gradient $-1 / 2$ in the double-logarithmic plot in panel (b). This means that $\mathrm{d} x_{0.02} / \mathrm{d} t \approx c_{1} t^{-1 / 2}$ and thus $x_{0.02} \approx 2 c_{1} t^{1 / 2}+c_{2}$ for large $t$, where $c_{1}(>0)$ and $c_{2}$ are some constants depending on $\mathrm{M}$ and $\mathrm{K}$. Therefore, we may conclude that the high-temperature region formed near the plate diffuses away from the plate, its width increasing in proportion to $t^{1 / 2}$.

We also try to measure the rate of decrease of the local peak at the wave front of streaming motion (or the weak compression wave). Let us denote by $\Theta(t)$ the value of $\bar{T}\left(x_{1}, t\right)-1$ at the local peak of the wave front [i.e., the height of the peak in Fig. 3(f)]. Figure 5(a) is the double-logarithmic plot of $\Theta(t)$ vs $t$, and the gradients of the curves, $\alpha(\Theta)=\mathrm{d} \log _{10} \Theta / \mathrm{d} \log _{10} t$, vs $t$ are plotted in Fig. 5(b). The figure shows a tendency that the gradients approach a constant value between -0.16 and -0.2 , but we need further computation until much later times to draw some

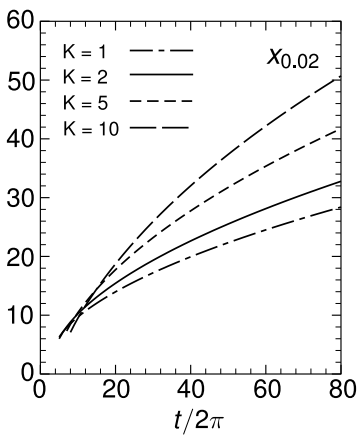

(a)

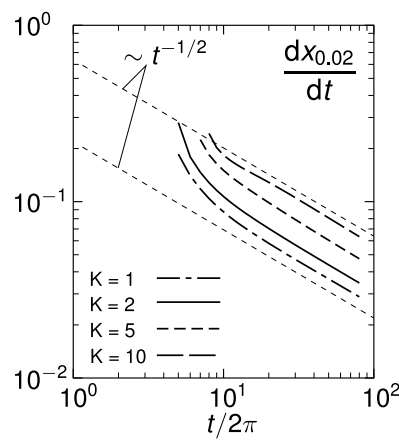

(b)

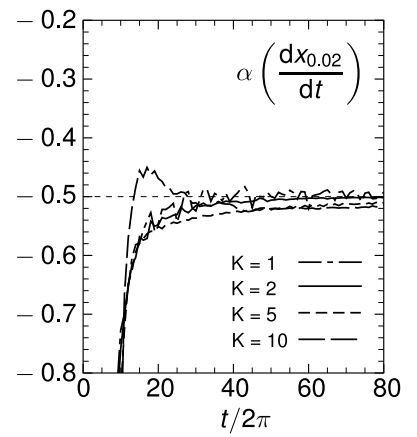

(c)

FIGURE 4. Time evolution of $x_{0.1}, \mathrm{~d} x_{0.1} / \mathrm{d} t$, and $\alpha\left(\mathrm{d} x_{0.02} / \mathrm{d} t\right)$ for $\mathrm{M}=0.2$. (a) $x_{0.1}$ vs $t$, (b) $\mathrm{d} x_{0.1} / \mathrm{d} t$ vs $t$ (in the doublelogarithmic scale), (c) $\alpha\left(\mathrm{d} x_{0.02} / \mathrm{d} t\right)$ vs $t$. The results for $\mathrm{K}=1$ (dash-dotted), 2 (solid), 5 (dashed), and 10 (long-dashed) are shown. 


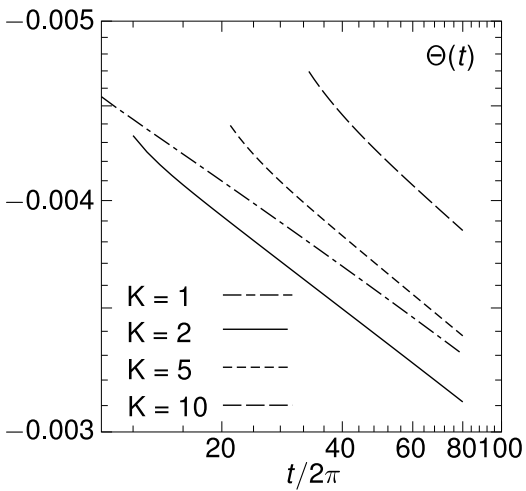

(a)

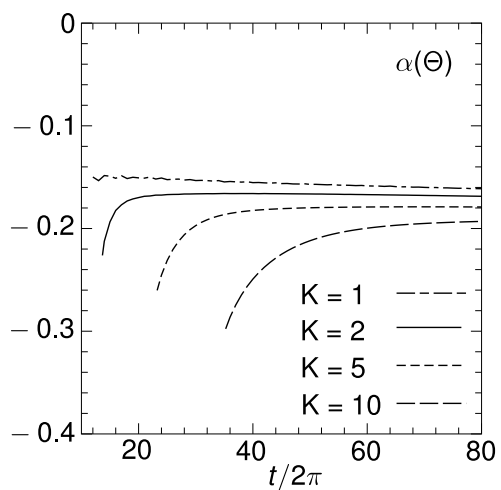

(b)

FIGURE 5. Time evolution of $\Theta$ and $\alpha(\Theta)$ for $\mathrm{M}=0.2$. (a) $\Theta$ vs $t$ (in the double-logarithmic scale), (b) $\alpha(\Theta)$ vs $t$. The results for $\mathrm{K}=1$ (dash-dotted), 2 (solid), 5 (dashed), and 10 (long-dashed) are shown.

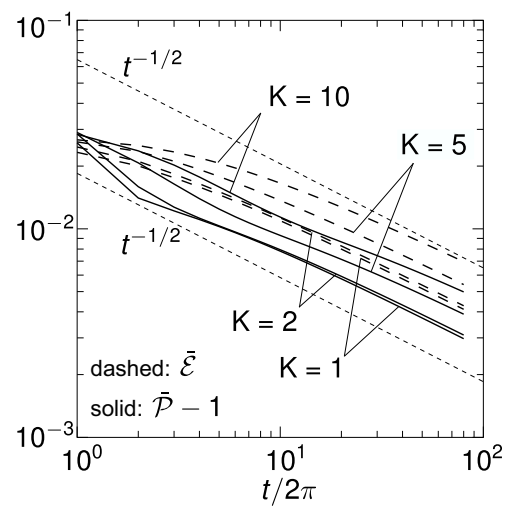

(a)

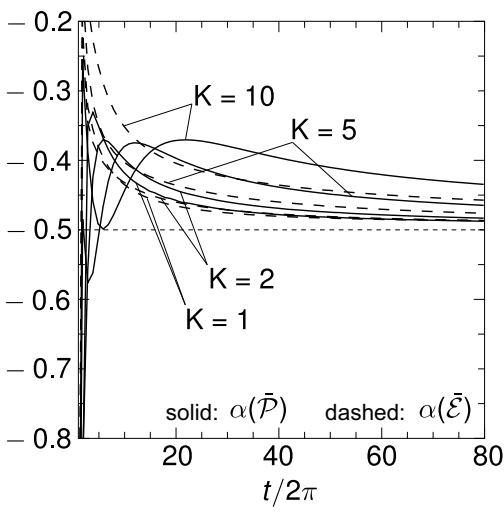

(b)

FIGURE 6. Time evolution of $\overline{\mathscr{P}}, \overline{\mathscr{E}}, \alpha(\overline{\mathscr{P}})$, and $\alpha(\overline{\mathscr{E}})$ for $\mathrm{M}=0.2$. (a) $\overline{\mathscr{P}}$ (solid) and $\overline{\mathscr{E}}$ (dashed) vs $t$ (in the double-logarithmic scale), (b) $\alpha(\overline{\mathscr{P}})$ (solid) and $\alpha(\overline{\mathscr{E}})$ (dashed) vs $t$. The results for $\mathrm{K}=1,2,5$, and 10 are shown.

conclusion. Within the information provided by Fig. 5, we may say that the decay of the peak is very slow and its asymptotic decay rate is something like $\Theta \propto 1 / t^{\gamma}$ with $\gamma$ around 0.2 .

\section{One-period Averages of Momentum and Energy Fluxes on the Plate}

Let us denote by $\overline{\mathscr{P}}$ and $\overline{\mathscr{E}}$ the time averages (over one period) of $\mathscr{P}$ and $\mathscr{E}$ [Eq. (11)], the $X_{1}$ component of the momentum and the energy, per unit area and time, transferred to the gas from the oscillating plate. More precisely, they are defined by

$$
[\overline{\mathscr{P}}(t), \overline{\mathscr{E}}(t)]=\frac{1}{2 \pi} \int_{t-2 \pi}^{t}[\mathscr{P}(s), \mathscr{E}(s)] \mathrm{d} s
$$

If a periodic state is established near the plate, $\overline{\mathscr{P}}(t)-1$ and $\overline{\mathscr{E}}(t)$ vanish because of the periodicity. We now investigate the time evolution of $\overline{\mathscr{P}}(t)$ and $\overline{\mathscr{E}}(t)$. Figure 6(a) is the plot of $\overline{\mathscr{P}}(t)$ and $\overline{\mathscr{E}}(t)$ vs $t$ in double logarithmic scale for $\mathrm{M}=0.2$ and for some different values of K. Figure 6(b) shows the gradients of the curves in Fig. 6(a), i.e., $\alpha(\overline{\mathscr{P}})=\mathrm{d} \log _{10}(\overline{\mathscr{P}}-1) / \mathrm{d}^{\log _{10}} t$ and $\alpha(\overline{\mathscr{E}})=\mathrm{d} \log _{10} \overline{\mathscr{E}} / \mathrm{d} \log _{10} t$, as functions of $t$. We must say that our computation up to $t / 2 \pi=80$ is not enough, in particular for large $\mathrm{K}$, to draw some definite conclusions. Nevertheless, it is seen 
from Figs. 6(a) and 6(b) that $\overline{\mathscr{P}}(t)-1$ and $\overline{\mathscr{E}}(t)$ tend to vanish as time goes on and that the curves in Fig. 6 tend to approach straight lines with gradient $-1 / 2$. In other wards, as time proceeds, $\overline{\mathscr{P}}(t)-1$ and $\overline{\mathscr{E}}(t)$ tend to vanish in such a manner that $\overline{\mathscr{P}}-1 \approx c_{p} t^{-1 / 2}$ and $\overline{\mathscr{E}} \approx c_{e} t^{-1 / 2}$ with positive constants $c_{p}$ and $c_{e}$. This provides a piece of numerical evidence that the gas near the plate approaches steady oscillation.

\section{CONCLUDING REMARKS}

In the present study, we have investigated numerically the nonlinear acoustic wave propagation in a semi-infinite expanse of a rarefied gas, caused by an infinitely wide plate that starts longitudinal oscillation, on the basis of the BGK model of the Boltzmann equation and the diffuse reflection boundary condition on the plate. An emphasis is placed on accurate description of the discontinuities in the molecular velocity distribution function in the gas that is produced continuously by the oscillating plate (though the behavior of the velocity distribution function is omitted in the paper because of the limited space). The resulting macroscopic quantities show that a weak compression wave propagates toward infinity (streaming motion) and decays very slowly. It is also shown that a high-temperature region is formed near the oscillating plate and diffuses slowly toward infinity and that the gas near plate approaches a periodic state slowly, the rate of approach being more or less proportional to $t^{-1 / 2}$.

\section{ACKNOWLEDGMENTS}

The authors thank Professors Takeru Yano and Shigeru Takata for valuable discussions. This work was supported by the Grants-in-Aid for Scientific Research No. 23360048 and No. 24·2418 from JSPS.

\section{REFERENCES}

1. L. D. Landau and E. M. Lifshitz, Fluid Mechanics, Pergamon Press, Oxford, 1959.

2. Y. Sone, Molecular Gas Dynamics: Theory, Techniques, and Applications, Birkäuser, Boston, 2007.

3. L. Sirovich and J. K. Thurber, J. Acoust. Soc. Am. 37, 329-339 (1965).

4. H. Grad, J. SIAM Appl. Math. 14, 935-955 (1966).

5. J. R. Thomas, Jr. and C. E. Siewert, Trans. Theory and Stat. Phys. 8, 219-240 (1979).

6. S. K. Loyalka and T. C. Cheng, Phys. Fluids 22, 830-836 (1979).

7. N. G. Hadjiconstantinou and A. L. Garcia, Phys. Fluids 13, 1040-1046 (2001).

8. F. Sharipov and W. Marques, and G. M. Kremer, J. Acoust. Soc. Am. 112, 395-401 (2002).

9. R. D. M. Garcia and C. E. Siewert, Z. Angew. Math. Phys. 57, 94-122 (2006).

10. F. Sharipov and D. Kalempa, J. Acoust. Soc. Am. 124, 1993-2001 (2008).

11. P. L. Bhatnagar, E. P.Gross, and M. Krook, Phys. Rev. 94, 511-525 (1954).

12. P. Welander, Ark. Fys. 7, 507-553 (1954).

13. Y. Inoue and T. Yano, J. Acoust. Soc. Am. 94, 1632-1642 (1993).

14. T. Yano and Y. Inoue, Phys. Fluids 8, 2537-2551 (1996).

15. T. Ohwada and M. Kunihisa, in Rarefied Gas Dynamics, edited by A. D. Ketsdever and E. P. Muntz, AIP, Melviele, 2003, pp. 202-209.

16. C. K. Chu, Phys. Fluids 8, 12-22 (1965).

17. T. Sone and S. Takata, Transp. Theor. Stat. Phys. 21, 501-530 (1992).

18. T. Tsuji and K. Aoki, in Rarefied Gas Dynamics, edited by D. A. Levin, I. Wysong, A. L. Garcia, and L. A. Gochberg, AIP, Melville, 2011, pp. 140-145.

19. T. Tsuji and K. Aoki, J. Stat. Phys. 146, 620-645 (2012).

20. T. Tsuji and K. Aoki, in preparation.

21. K. Aoki, Y. Sone, K. Nishino, and H. Sugimoto, in Rarefied Gas Dynamics, edited by A. E. Beylich, VCH, Weinheim, 1991, pp. 222-231. 
Copyright of AIP Conference Proceedings is the property of American Institute of Physics and its content may not be copied or emailed to multiple sites or posted to a listserv without the copyright holder's express written permission. However, users may print, download, or email articles for individual use. 